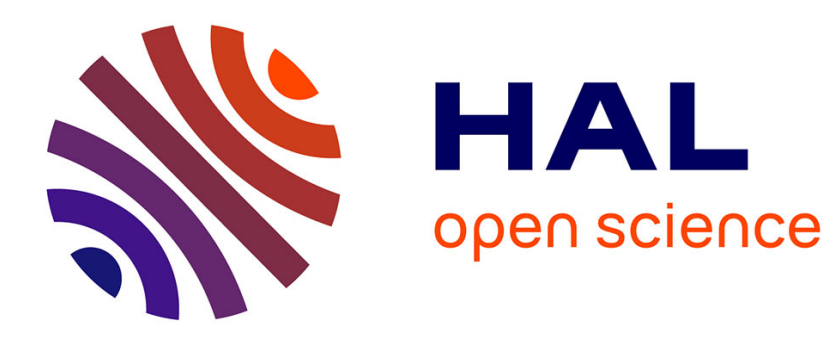

\title{
Salammbô - une mythologie du vivant
}

Gisèle Séginger

\section{To cite this version:}

Gisèle Séginger. Salammbô - une mythologie du vivant. Modern Language Notes, 2013, Salammbô, 150 years, 128 (4), pp.694-712 10.1353/mln.2013.0052 . hal-01305051

\section{HAL Id: hal-01305051 \\ https://hal.science/hal-01305051}

Submitted on 20 Apr 2016

HAL is a multi-disciplinary open access archive for the deposit and dissemination of scientific research documents, whether they are published or not. The documents may come from teaching and research institutions in France or abroad, or from public or private research centers.

$$
\text { Copyright }
$$

L'archive ouverte pluridisciplinaire HAL, est destinée au dépôt et à la diffusion de documents scientifiques de niveau recherche, publiés ou non, émanant des établissements d'enseignement et de recherche français ou étrangers, des laboratoires publics ou privés. 


\title{
Salammbô - une mythologie du vivant
}

\author{
Gisèle Séginger
}

Journal: $\underline{M L N}$

Volume 128, Number 4, September 2013 (French Issue)

pp. 694-712 | 10.1353/mln.2013.0052

L'étrangeté de Salammbô provient en partie de la réécriture des mythes et des symboles. Ils sont présents à tous les niveaux de l'intrigue, et ils contribuent donc à la cohérence du roman. Les couleurs, les métaux, les végétaux se répondent et tissent un réseau de significations religieuses. Cette harmonie donne une présence exceptionnelle aux images inventées par Flaubert, et elle crée une sorte d'illusion de réalité, parce que dans cet univers inventé tout se correspond. L'étrangeté - parfois presque onirique comme dans l'épisode du temple de Tanit au chapitre $\mathrm{V}$ - acquiert ainsi une présence et une force qui sont en principe celles d'un monde réel bien représenté. Flaubert trouve en outre dans les mythes qu'il manipule et fusionne de manière syncrétiste l'idée de péripéties qu'il développe dans la fiction. Il inscrit aussi les mythes dans la spatialité romanesque, dans le décor, dans la vie des personnages qui sont agis par la force des dieux, et enfin dans l'histoire non dialectique d'une guerre qui oppose la vie et la mort, comme Tanit et Moloch. Toutefois malgré cette saturation du récit par le conflit mythique entre les deux dieux qui sont aussi deux principes et deux pulsions le roman ne se réduit pas à ce dualisme schématique. Flaubert réinvente les mythes par un travail de stratification qui superpose des mythes d'origine diverses et la condensation des symboles et des significations empruntés à des religions différentes donne une profondeur et un mystère à cette religion réinventée qui semble faire signe vers une multitude de mythes. De surcroit, Flaubert troue de silences ces mythes recréés par la fiction, en dispersant leurs éléments, en effaçant des significations, souvent développées dans les brouillons. L'exemple de l'épisode du temple de Tanit en fait la preuve : Flaubert incarne dans la structure de l'édifice et dans son décor symbolique des croyances cosmogoniques. Il évite toutefois d'en dévoiler la signification complète et cohérente dans le texte définitif. C'est ainsi que certaines images gagnent leur radicale étrangeté. Flaubert a éliminé le chapitre explicatif qu'il avait pourtant intégralement rédigé - et qu'il avait prévu à un moment de placer au début du roman - probablement parce que les analyses de l'esprit du peuple carthaginois et de sa religion auraient trop éclairé le symbolisme religieux du roman, et en particulier les éléments mythiques qu'il met en scène dans l'épisode du temple de Tanit, l'un des épisodes qui tend le plus vers l'onirisme érudit et symbolique.

Ce temple est aussi insolite parce que Flaubert utilise la focalisation interne pour que le lecteur découvre le sanctuaire par les yeux de deux personnages et non par l'intermédiaire d'une description assumée par un narrateur omniscient. Le temple de Tanit est vu par deux étrangers qui ne connaissent pas parfaitement ce culte carthaginois, ce qui préserve l'étrangeté du lieu. Ils en savent tout de même assez pour en comprendre certains éléments et, dans le cas de Mâtho pour redouter l'action de la déesse, et vivre une expérience presque hallucinatoire.

Pour construire la théogonie et la cosmogonie carthaginoises, selon une méthode bien rodée depuis La Tentation de saint Antoine de 1849, Flaubert a lu de nombreux ouvrages, et il réutilise tout particulièrement l'ouvrage de Creuzer qu'il avait découvert à l'occasion de la rédaction de La Tentation: Religions de l'antiquité considérées principalement dans leur forme 
symbolique et mythologique ${ }^{1}$. Creuzer souligne une particularité des connaissances sur la religion phénicienne qui pouvait être comme une invitation à la fiction: "nous sommes ici d'une indigence extrême en fait de documents [...]. C'est de la quatrième main seulement que nous tenons les lambeaux de la mythologie phénicienne ${ }^{2}$.» En effet, un soi-disant Phénicien, Sanchionathon, plusieurs siècles avant Jésus-Christ aurait écrit une Histoire phénicienne perdue, qui aurait été traduite en grec par un certain Philon de Byblos au $\mathrm{II}^{\mathrm{e}}$ siècle après $\mathrm{J}$. C. Ce texte également perdu - aurait été utilisé par Porphyre contre le christianisme dans une œuvre qui n'a pas davantage été conservée ; Eusèbe de Césarée, un chrétien cette fois, a extrait de Porphyre une partie de la traduction de Philon de Byblos, qu'il donne dans son œuvre intitulée Préparation évangélique. Celle-ci est la seule qui nous soit parvenue. Les circonstances pouvaient plaire à Flaubert : le mythe cosmogonique phénicien tel qu'il est connu au $\mathrm{XIX}^{\mathrm{e}}$ siècle - et relaté encore par Creuzer - a été passé au filtre d'une série de pensées divergentes: Philon (Phénicien de langue grecque, fils adoptif d'un consul romain) était athée et évhémériste ; Porphyre était un néo-platonicien et Eusèbe un chrétien. De surcroît, le texte de Sanchionathon a donné lieu en 1836 à une supercherie : la publication par Wagenfeld de neuf livres de Sanchionathon qu'il prétendait avoir découverts et dont il voulait accréditer l'authenticité ${ }^{3}$. Ces livres apocryphes ont été néanmoins traduits en français et font partie des sources de Flaubert.

Sans doute ces manipulations déjà subies par la cosmogonie phénicienne pouvaient-elles stimuler la rêverie érudite de Flaubert. Dans les manuscrits, le retraitement fictionnel de cette cosmogonie dans l'épisode du temple de Tanit ébauche une vision du monde et de la création insolite, tout à la fois antispiritualiste et antimatérialiste, et surtout dynamique, voire évolutionniste. Ainsi Flaubert met-il en abyme dans ce chapitre une logique qui structure par ailleurs l'ensemble du roman.

J'aborderai trois points : 1) le rapport de l'épisode du temple de Tanit avec celui des animaux fantastiques de La Tentation : cet épisode de Salammbô témoigne d'un nouvel ancrage épistémologique des questionnements de Flaubert; 2) la construction d'une mythologie naturaliste dans les brouillons de l'épisode 3) l'étrangeté et la dimension fantastique de cette mythologie du vivant.

\section{Un ancrage épistémologique entre antiquité et modernité}

Depuis les années 1840 et la lecture de l'Esthétique de Hegel, Flaubert s'interroge sur les formes fantastiques. Dans l'Éducation sentimentale de 1845, l'artiste Jules expliquait le fantastique comme une «surabondance de l'élément moral ${ }^{4}$ » et s'il en comprenait ainsi l'importance dans l'histoire de l'art, et dans certaines œuvres comme le Faust de Goethe, il en faisait néanmoins la critique : «on ne fera rien de beau en inventant des animaux qui ne sont pas, des plantes qui n'existent point, en donnant des ailes à un cheval, des queues de poisson à des corps de femmes, révélations d'un type insaisissable, rêves sans corps ${ }^{5}$ ». Quelques mois après, Flaubert rédige pourtant une première Tentation. Mais le fantastique est versé au compte d'un

${ }^{1}$ Frédéric Creuzer, Religions de l'antiquité. L'ouvrage a été traduit et refondu en partie par J.-D. Guigniaut, avec la collaboration d'Alfred Maury et E. Vignet (Paris : Treuttel et Würtz, 1825-1851) 4 tomes en 10 volumes.

${ }^{2}$ Creuzer, Religions de l'antiquité II, 1, p. 9-10.

${ }^{3}$ Friedrich Wagenfeld, Analyse des neuf livres de la chronique de Sanchoniaton, traduit de l'allemand par P. Lebas (Paris, 1836).

${ }^{4}$ Gustave Flaubert, L'Éducation sentimentale, Euvres de jeunesse, sous la direction de Claudine GothotMersch et Guy Sagnes, Euvres complètes (Paris : Gallimard, Bibliothèque de la Pléiade, 2001)1 : 1039.

${ }^{5}$ Flaubert, L'Éducation 1038. 
ermite halluciné. Surabondance des désirs, échec de la sublimation religieuse : les bêtes monstrueuses incarnent des pulsions irrépréhensibles, qui sont en rapport avec les sept Péchés capitaux. En 1856, avant de rédiger Salammbô, Flaubert reprend La Tentation. Il conserve l'hallucination des bêtes fantastiques sans changement majeur, sans y introduire d'êtres réels et vivants. Toutefois, en voyant les animaux, Antoine sent la vie qui lui grouille au ventre et il voudrait fusionner avec la nature entière :

J'ai besoin d'aboyer, de beugler, de hurler ! je voudrais vivre dans un antre, souffrir de la fumée, porter une trompe, tordre mon corps, - et me diviser partout, être en tout, m'émaner avec les odeurs, me développer comme les plantes, vibrer comme le son, briller comme la lumière, me blottir sous les formes, pénétrer chaque atome, circuler dans la matière, être matière moi-même pour savoir ce qu'elle pense. $^{6}$

Atome, matière, formes : cette tentation diabolique est panthéiste et matérialiste. Du point de vue épistémologique, elle se rattache à une vulgate pythagoricienne constituée dans les années 17701820 grâce à la Philosophie de la nature de Delisle de Sales, puis à la publication des Vers dorés de Pythagore par Fabre d'Olivet (1813). On trouve les traces de cette vulgate dans le Faust de Goethe et dans le poème de Nerval «Vers dorés », publié en mars 1845 dans L'Artiste (sous le titre «Pensée antique »), puis repris dans Les Chimères (1856). Nerval résumait l'essentiel du pythagorisme tel qu'on le concevait alors. La matière est pensante :
À la matière même un verbe est attaché
$[\ldots]$
Un pur esprit s'accroît sous l'écorce des pierres ${ }^{7}$ !

Par contre, dans La Tentation de 1874 Flaubert intègrera une interrogation plus moderne sur l'origine de la vie et l'histoire des êtres organisés qu'il reprendra encore dans Bouvard et Pécuchet bien que sous une autre forme : il ébauchera alors sur un mode ironique une série de mythologies scientifiques de la création, dévoilant au cœur de la science l'étrange et le fantastique. L'ancrage épistémologique de ces deux dernières œuvres s'est déplacé.

Or, entre les Tentations de 1849-1856 et les deux derniers romans sur les croyances et les savoirs, Salammbô constitue un tournant: le questionnent de Flaubert se transforme et son soubassement épistémologique n'est plus le même. Dans la cosmogonie de Salammbô, Flaubert réécrit en termes mythologiques des idées qui appartiennent aux problématiques du XIX ${ }^{\mathrm{e}}$ siècle et même quelques-unes de ses propres réflexions qu'il confiait à ses amis dans ses lettres dès les années 1850. C'est une période où il lit des ouvrages de sciences naturelles, tandis que son ami Louis Bouilhet prépare son grand poème scientifique de 1854 : Les Fossiles. Ce poème sur les âges du monde, est en partie inspiré par Cuvier, avec toutefois une différence majeure puisqu'il raconte l'origine du monde, de la vie et des espèces sortant de l'élément humide (ce qu'évitait Cuvier), et le poème esquisse une perspective évolutionniste. Or, c'est dans cette même période,

${ }^{6}$ Gustave Flaubert, La Tentation de saint Antoine (1856), éd. G. Séginger, Euvres complètes, sous la direction de Claudine Gothot-Mersch (Paris : Gallimard, Bibliothèque de la Pléiade, 2001)II, p. 99.

${ }^{7}$ Il n'est pas impossible que Flaubert ait lu le poème de Nerval. Tous deux avaient pour ami commun Gautier, et le poème est paru dans une revue à laquelle Flaubert - à la demande de Gautier - confiera la publication des premiers fragments de La Tentation. 
que Flaubert commence à citer dans sa correspondance des naturalistes comme Buffon et l'évolutionniste Geoffroy Saint-Hilaire. Il vante la beauté des sciences naturelles, modèles pour l'histoire et pour la littérature, par leur impassibilité et leur sens de l'infini. En 1853, il oppose la méthode de Geoffroy Saint-Hilaire aux erreurs des dualismes philosophiques qui font obstacle à l'esprit scientifique : «Les matérialistes et les spiritualistes empêchent également de connaître la matière et l'esprit, parce qu'ils scindent l'un et l'autre ${ }^{8}$. » En 1859, tandis qu'il rédige Salammbô, il revient encore sur ce travers :

Je suis convaincu que les appétits matériels les plus furieux se formulent insciemment par des élans d'idéalisme, de même que les extravagances charnelles les plus immondes sont engendrées par le désir pur de l'impossible, l'aspiration éthérée de la souveraine joie. Et d'ailleurs je ne sais (et personne ne sait) ce que veulent dire ces deux mots : âme et corps, où l'une finit, où l'autre commence. Nous sentons des forces et puis c'est tout'?

Le débat stérile entre le matérialisme et spiritualisme est remis en jeu dans Salammbô grâce à des croyances cosmogoniques carthaginoises qui présentent l'avantage d'éluder le dualisme en esquissant un vitalisme étrange, qui sera par la suite - dans La Tentation de 1874 - facile à retraiter dans le sens d'un échange dialogique entre la vie et la mort.

L'opposition entre Moloch et Tanit ressemble à l'antagonisme de la Luxure et de la Mort dans les deux premiers textes de La Tentation. Quant au Diable, il montre à Antoine dans le défilé des dieux Milytha «couverte de ses voiles ${ }^{10}$ » et Moloch, furieux «crachant des flammes par les narines et dont le ventre, bourré d'hommes, hurle comme une forêt incendiée ${ }^{11} »$. Mais dans La Tentation, la destruction et le Diable l'emportaient toujours. Au contraire, dans ses ébauches préparatoires pour Salammbô, Flaubert met au point une autre logique : « la vie et la mort - d'une génération incessante et d'une destruction sans fin travaillant ensemble quoique ennemies - antagonisme qui était un éternel hymen ${ }^{12} »$. Ailleurs pour défendre cette idée, il se réfère à Héraclite, à l'idée philosophique d'une unité dynamique qui naît de l'opposition : «Moloch. Quatre aspects : planète - Feu - créateur suprême - roi. Phta dieu du feu tient un œuf dans sa bouche. Tout meurt pour l'accomplissement de l'unité et tout renaît en elle. Au sein de l'unité, il faut que la multiplicité éclate - toute chose naît de l'opposition. Il n'y aurait pas de génération sans la guerre (Héraclite) ${ }^{13} »$. Enfin, dans un brouillon, il revient sur l'idée de «force »: « la mort et la vie, la destruction et l'enfantement, la douleur et la joie, tout s'égalisait, tout s'absorbait, dans le feu, dans une force suprême infinie, inaccessible à la pitié et indifférente à la vertu ${ }^{14}$. » La notion de «force » est capitale lorsqu'il s'agit de dépasser à la fois le matérialisme et le spiritualisme, afin de penser non plus dans le cadre métaphysique des vieux dualismes mais dans la perspective d'une conception plus moderne de l'infini et du mouvement. La notion de force permet à Flaubert de se débarrasser à la fois de l'idée de finalité (défendue par les spiritualistes) et de l'idée du progrès, défendue par les positivistes. Cette notion de force sera importante dans l'évolutionnisme spencérien qui refusera également l'opposition du

\footnotetext{
${ }^{8}$ Lettre à Louise Colet du 7 juillet 1853.

${ }^{9}$ Lettre à Mlle Leroyer de Chantepie du 18 février 1859.

${ }^{10}$ Flaubert, Tentation 131.

${ }^{11}$ Flaubert, Tentation 131.

${ }^{12}$ N.a.fr. $23658, \mathrm{f}^{\circ} 135 \mathrm{v}^{\circ}$.

13 N.a.fr. $23660, \mathrm{f}^{\circ} 98$.

${ }^{14}$ N.a.fr. $23658, \mathrm{f}^{\circ} 163 \mathrm{v}^{\circ}$
} 
matérialisme et du spiritualisme ${ }^{15}$. Mais la réception française de la philosophie de Spencer date des années 1870, et Flaubert lui-même ne l'a probablement lu qu'à la fin des années 1870, tandis qu'il préparait Bouvard et Pécuchet ${ }^{16}$. Par contre, il connaissait bien Félix Pouchet, naturaliste rouennais et défenseur de la génération spontanée dans les années $1850^{17}$. Celui-ci émettait l'hypothèse athéiste de l'existence d'une «force plastique » à l'œuvre dans le monde. La célèbre controverse qui opposa Pasteur et Pouchet éclata en 1859 après la publication par le second d'un livre intitulé Hétérogénie ou Traité de la génération spontanée. Flaubert ne lira l'ouvrage qu'en 1860 après la rédaction de son chapitre sur le temple de Tanit ${ }^{18}$, mais il connaissait probablement d'autres travaux similaires du naturaliste rouennais, qu'il fréquentait d'ailleurs, étant un ami de la famille. Il était plus particulièrement lié avec son fils Georges qui avait aussi évoqué la théorie de la génération spontanée dans son livre de 1858, De la pluralité des races humaines (offert à Flaubert $)^{19}$, et qui conseillait Flaubert sur les questions de botanique pendant la rédaction de Salammbô.

La notion de force intéresse sans doute aussi Flaubert parce qu'elle appartient à plusieurs théories. On la trouve en particulier chez Héraclite (cité dans les manuscrits de Salammbôo $^{20}$ ). Elle est centrale dans sa pensée : selon Héraclite le monde tient sa cohésion du mouvement des forces qui s'équilibrent et évitent sa chute dans le néant ainsi que la dispersion des éléments. L'origine est aussi conçue par Héraclite comme une force : c'est le feu, créateur de toute chose. Le feu est selon lui un élément immatériel et comme abstrait, et en cela capable d'impulser le mouvement. Significativement dans la troisième salle du temple de Tanit, où se trouve le voile magique, on voit un autel sur lequel brûle des flammes, et le zaïmph (symbole du mystère de l'univers) est suspendu derrière ce feu sacré.

Dans le chapitre III, au début de la cosmogonie, racontée par Schahabarim à Salammbô se trouve le souffle, qui possède des qualités similaires, et qui permet de faire l'économie d'une création divine. Flaubert laisse alors au grand prêtre la responsabilité d'une mise en cause de l'idée de création divine. Frustré dans son désir pour la jeune fille, et tourmenté par sa rancœur à l'égard d'une divinité qui lui a ôté sa virilité, l'eunuque éprouve une infinie jouissance à déconstruire l'idée d'une Tanit toute-puissante. Selon lui, les dieux ne sont que des créations tardives. À l'imagination étriquée de Salammbô qui conçoit sa déesse de manière anthropomorphique, dotée d'une volonté humaine, il oppose une vision poétique et impersonnelle de la création. Il imagine des processus purement physiques, une sorte d'évolution, puis un Big Bang à l'antique, avec l'explosion de l'œuf cosmogonique :

Avant les Dieux, les ténèbres étaient seules, et un souffle flottait, lourd et indistinct comme la conscience d'un homme dans un rêve. Il se contracta, créant le Désir et la Nue, et du Désir et de la Nue sortit la Matière primitive. C'était une eau bourbeuse, noire, glacée, profonde. Elle enfermait des monstres insensibles, parties

${ }^{15}$ Le mot est récurrent chez Spencer dès la publication en 1855 des Principes de psychologie, puis dans Premiers principes (1862), ouvrage traduit en français en 1871.

${ }^{16}$ Il cite alors dans sa documentation De l'éducation, intellectuelle et morale (g 226, vol. 8, $\left.\mathrm{f}^{\circ} 40 \mathrm{r}^{\circ}\right)$. La première occurrence dans la Correspondance date d'une lettre à Mme Roger des Genette de janvier 1878 .

${ }^{17}$ En 1858, Félix Pouchet publie deux articles dans les Comptes rendus de l'Académie des sciences pour prouver la validité de sa thèse sur la génération spontanée.

${ }^{18}$ Lorsqu'il lit l'Hétérogénie, en août 1860, il en est déjà à la rédaction du chapitre VIII.

${ }^{19}$ Flaubert possédait l'ouvrage avec une dédicace de Georges Pouchet. Voir La Bibliothèque de Flaubert, sous la direction d'Yvan Leclerc, Publications de l'université de Rouen, p. 2001, p. 174.

${ }^{20}$ N.a.fr. $23660, f^{\circ} 98$. 
incohérentes des formes à naître et qui sont peintes sur la paroi des sanctuaires.

Puis la Matière se condensa. Elle devint un œuf. Il se rompit. Une moitié forma la terre, l'autre le firmament. Le soleil, la lune, les vents, les nuages parurent; et, au fracas de la foudre, les animaux intelligents s'éveillèrent. Alors Eschmoûn se déroula dans la sphère étoilée ; Khamon rayonna dans le soleil ; Melkarth, avec ses bras, le poussa derrière Gadès; les Kabyrim descendirent sous les volcans, et Rabbetna, telle qu'une nourrice, se pencha sur le monde, versant sa lumière comme un lait et sa nuit comme un manteau ${ }^{21}$.

Le manteau de Tanit (zaïmph) est comme l'enveloppe de mystère qui couvre l'univers.

Pour concevoir le récit cosmogonique de Schahabarim Flaubert a utilisé le texte des Religions de l'antiquité qui lui fournira aussi des éléments symboliques pour la représentation de Tanit au chapitre V :

Le Temps, le Désir et la Nue étaient [...] les trois grands principes de toutes choses, selon les Sidoniens. De l'union des deux derniers naquirent l'Ether ou l'air mâle, et Aura ou l'air femelle, qui à leur tour produisirent un œuf. Chez Eusèbe, le Souffle de l'esprit ou le Vent primitif, et la Nuit primitive, figurent comme principe des choses. Sanchionathon connaît aussi Môt ou le limon primitif. De là certains animaux d'abord dépourvus de sentiment, puis doués d'intelligence ; de là le soleil, la lune et les étoiles.

[...] Ici, comme dans le système égyptien, on voit l'esprit et la matière tous deux incréés et préexistants. Môt est un nom d'Isis pris dans son sens élevé. ${ }^{22}$

Comme le signale Creuzer par ailleurs, la cosmogonie phénicienne contredit l'idée judéochrétienne d'une création divine. L'esprit et la matière tous deux incréés préexistent; Isis (à laquelle Tanit sera explicitement associée dans les brouillons de Flaubert) est identifiée par Creuzer à Môt le limon primitif d'où sont sortis tous les animaux, le soleil, la lune, les étoiles. Le démiurge, ou dieu ouvreur, Phta en égyptien, n'arrive que tardivement. Son action et son pouvoir sont réduits ; il se contente de briser l'œuf du monde « en deux parties dont l'une forme le ciel et l'autre la terre ${ }^{23}$.»

Creuzer signale également qu'en Phénicie et en Syrie comme en Égypte, les dieux ont souvent les deux sexes : ils sont androgynes. Il donne l'exemple d'Isis « passive vis-à-vis du taureau générateur, du soleil fécondant; active vis-à-vis de la terre qu'elle féconde à son tour, en lui communiquant les germes producteurs qu'elle a reçus». Elle est alors figurée comme «la mère universelle des êtres ${ }^{24} »$. Dans ce cas, explique Creuzer, elle incarne l'idée fréquente en Haute-Asie d'une « toute-puissance divine se suffisant à elle-même ${ }^{25}$ ».

Flaubert utilise tous ces éléments à la fois pour écrire la cosmogonie racontée par Schahabarim qui relègue très bas Tanit (chapitre III), mais aussi pour écrire le contraire dans le chapitre V lorsqu'il représente Tanit comme une déesse-Univers toute-puissante, omniféconde, hermaphrodite et barbue.

\footnotetext{
${ }^{21}$ Gustave Flaubert, Salammbô, éd. établie par G. Séginger (Paris : Flammarion, « GF », 2001$) 111$.

${ }^{22}$ Creuzer, Religions de l'antiquité II, 1, p. 12-14.

${ }^{23}$ Creuzer, Religions de l'antiquité II, 1, p. 14.

${ }^{24}$ Creuzer, Religions de l'antiquité r II, 1, p. 4.

${ }^{25}$ Creuzer, Religions de l'antiquité II, 2, p. 5.
} 
Au chapitre III, Schahabarim expose l'auto-engendrement de l'univers par trois processus impersonnels: contraction (contraction du souffle), condensation (de la matière), éclosion/explosion (de l'œuf). Pas de dieu ouvreur, l'œuf éclot seul. Par contre dans le chapitre $\mathrm{V}$, dans l'une des salles du temple de Tanit, on voit un immense corps de femme qui remplit toute la pièce ; un œuf énorme pend à un fil de son nombril. Et, dans cet épisode, un peu plus loin, Tanit apparait comme la cause de tout. N'y a-t-il pas une contradiction entre les deux chapitres ? Oui, mais cette contradiction relève d'une poétique de l'exposition des croyances : le roman laisse coexister des points de vue différents, voire divergents, celui de l'eunuque châtré à cause de Tanit et qui est attiré par des croyances plus scientifiques, celui de Mâtho qui est amoureux et qui prend Salammbô pour Tanit et celle-ci pour la déesse créatrice de l'univers.

Dans Salammbô, l'étrangeté de la religion carthaginoise tient au tremblé des représentations : tandis que les mythes racontent pour expliquer et pour rationaliser, le récit flaubertien raconte pour ouvrir des failles dans la représentation.

\section{Des manuscrits au texte}

Des brouillons au texte, Flaubert réalise un travail inverse par rapport à celui des historiens des religions qu'il a lus. Ceux-ci expliquent et racontent les mythes en faisant des rapprochements syncrétistes pour dégager des symboles les plus étranges une signification, montrer sa formation dans l'histoire grâce à des échanges culturels. Flaubert au contraire fait des trous dans les récits mythiques, il procède par dispersion ou suppression des symboles trop lisibles, juxtaposition d'images plastiques évidées de leur signification.

Il avait d'abord prévu de placer dans l'enceinte du temple des «édicules de forme différente abritant des divinités étrangères en rapport avec Tanit, dédoublements de sa personne sous des appellations barbares et successivement admises dans son temple et sa pensée ${ }^{26}{ }$. Il renonce finalement à ces édicules annexes. Il pense alors représenter dans l'une des salles du temple les «tanits successives ${ }^{27}$ » avant d'abandonner aussi cette idée pour placer dans la seconde salle du temple une seule statue syncrétiste qui réunit les emblèmes d'autres divinités :

$\mathrm{Au}$ fond de la salle assise sur un char que traînaient des lions, La Rabetna suprême, la vraie Tanit, l'omniféconde, la dernière inventée réunissait les attributs des dieux barbares en rapport avec elle. Des écailles, des plumes, des fleurs et des oiseaux lui montaient jusqu'à la ceinture. Elle portait comme Cybèle des tours sur la tête, comme Minerve une gorgone sur la poitrine, comme Vénus des dauphins à ses pieds. Pour pendants d'oreilles, elle avait des cymbales d'argent qui lui battaient sur les joues. Ses yeux fixes vous regardaient et une pierre lumineuse enchâssée à son front, éclairait toute la salle en se reflétant au-dessus de la porte, dans des miroirs de cuivre. $^{28}$

Son char est celui de Cybèle et le brouillon explicite cette assimilation. Quant à l'idée d'une déesse panthée qui présente un assemblage d'attributs empruntés à des religions diverses, elle provient de

\footnotetext{
${ }^{26}$ N.a.fr. $23658, \mathrm{f}^{\circ} 429$.

${ }^{27}$ N.a.fr. $23658, \mathrm{f}^{\circ} 415$.

${ }^{28}$ N.a.fr. $23658, \mathrm{f}^{\circ} 452 \mathrm{v}^{\circ}$.
} 
la représentation d'Artémis d'Éphèse décrite dans Religions de l'antiquitét ${ }^{29}$.

Dans l'un des premiers folios, Flaubert donne une indication intéressante qui éclaire l'évolution de la déesse dans la suite des brouillons : «Tanit cabirique ${ }^{30} »$. Or, les Cabires, bien étudiés par Creuzer ${ }^{31}$, sont des divinités mystérieuses qui dominent les airs, la terre et les mers, et qui détiennent une force tellurique. Les brouillons suivants peuvent donc développer l'idée d'une déesse unique, faisant la synthèse de tout: c'est la divinité «des forces terrestres et des clartés sidérales $^{32} »$. De brouillon en brouillon, Tanit devient la force créatrice suprême, maîtresse du ciel et de la nature, l'origine de toutes choses, une force de vie. Flaubert construit alors l'épisode de la traversée du temple-- comme une expérience mystique de communion avec la force du monde qui présente des similitudes avec ce qu'il écrira dans le dénouement de La Tentation de 1874. Mâtho touche à l'origine de la vie, et il semble habité par une puissance qui en provient. Il se transforme lui-même en un dieu.

Je n'indique que les étapes principales des réécritures de l'expérience de Mâtho.

Flaubert avait d'abord imaginé une traversée d'enceintes successives jusqu'à la troisième. Il plaçait là une scène étrange avec des animaux monstrueux et effrayants dans la nuit :

C'était une large cour avec des dalles de couleurs. Un arbre énorme en occupait le milieu. Au pied des murs s'ouvraient des arcades ténébreuses comme des cavernes. Mais sur le bord de chacune à ras du sol un mufle de bête féroce apparaissait. Il y en avait d'étendues par terre et qui dormaient roulées en boule comme des chats. Spendius et Mâtho faisaient pour passer de grandes enjambées. Mais les bêtes l'une après l'autre se réveillèrent et les tigres bombaient leur dos, les ours grommelaient, les lions tournaient autour d'eux en les regardant. Ils entendaient venir le pas léger des grosses pattes velues avec le claquement des griffes posant sur les dalles. Et ils ne pouvaient plus avancer. Elles restaient là flairant leurs mains, se frottant contre leurs cuisses. Les prunelles rouges étincelaient dans l'ombre et les larges gueules craquaient avec un bruit d'os. [...] Spendius pâlissait, il se sentait comme épuisé par ces forces terribles circulant autour de lui. Mâtho cependant s'en apercevait à peine ${ }^{33}$.

Les bêtes ralentissent la marche de Mâtho et de Spendius. Ce dernier est paralysé de peur tandis que Mâtho ne s'aperçoit de rien et contemple « avec extase la coupole de la déesse toute en cuivre rouge qui apparaissait derrière le mur entre les branches ${ }^{34} »$.

Puis Flaubert décide de supprimer les bêtes féroces dans la cour. Mais sur les murs de la seconde salle du temple apparaissent alors des animaux étranges. Ces peintures montrent la naissance des êtres naturels, «toutes les bêtes de la création », dit un brouillon, «toutes les formes se trouvaient là, comme si le réceptacle des germes, crevant dans une éclosion soudaine, se fût vidé sur les murs de la salle ${ }^{35} »$. C'est l'origine du monde. La création explose sur les murs, les formes

${ }^{29}$ «L'idole arrivée à son point de perfection offre à nos regards une figure panthée, assemblage des attributs les plus divers, où semble se reproduire cette multitude de traits mythiques puisés dans les religions de la Haute-Asie, de la Scythie et de l'Égypte »(Creuzer, Religions de l'antiquité II, 1, p. 136).

${ }^{30}$ N.a.fr. $23658, f^{\circ} 415$.

${ }^{31}$ Creuzer, Religions de l'antiquité, II, 1, p. 275-92).

${ }^{32}$ N.a.fr. $23658, \mathrm{f}^{\circ} 459$.

${ }^{33}$ N.a.fr. $23658, f^{\circ} 430$ (les bêtes féroces dans la troisième enceinte du temple de Tanit).

${ }^{34}$ N.a.fr. $23658, \mathrm{f}^{\circ} 430$.

${ }^{35}$ N.a.fr. $23658, \mathrm{f}^{\circ} 469 \mathrm{v}^{\circ}$. 
semblent encore inachevées ou en pleine mutation :

tout à l'entour une infinité de bêtes monstrueuses efflanquées, haletantes et pêlemêle, les pattes s'appuyant sur les oreilles, les gueules apparaissant sous les ventres [...] les serpents avaient des pieds, les taureaux avaient des ailes. Des poissons parmi les feuillages becquettaient des fruits. Des fleurs s'épanouissaient dans la mâchoire des crocodiles et des éléphants la trompe levée passaient en plein azur orgueilleusement comme des aigles $[\ldots]^{36}$

Comme il le fera à nouveau à la fin de La Tentation de 1874, Flaubert met ici en scène le spectacle fantastique d'une force génésique à l'œuvre. Sur les murs, la profusion de formes étranges, hybrides suggère le pouvoir de transformation de la force de vie et l'instabilité des formes vivantes.

Dans la troisième et dernière salle se trouve le fameux voile de Tanit. Les brouillons élaborent progressivement la description d'un tissu sacré et merveilleux qui contient toute la création naturelle et théogonique. Tanit est la maîtresse de la Nature, du Ciel et des Dieux. On retrouve donc sur son voile des animaux et des Dieux.

Dans la cour à la place des bêtes féroces, pour préparer l'arrivée dans la seconde salle du temple et la vision du réceptacle des germes, Flaubert place des courtisanes endormies. Il crée alors une atmosphère lourde de sensualité qui flotte et qui envahit Mâtho, en l'affaiblissant, d'autant plus qu'il a peur du sacrilège, tandis que dans cette nouvelle conception de l'épisode Spendius n'a plus peur. Au cours de son travail, Flaubert a donc inversé les rôles, car il a décidé de mettre en scène la rencontre mystico-érotique de Mâtho et de Tanit, qui prépare la scène d'hiérogamie sous la tente avec Salammbô. Lorsque Mâtho pénètre dans la salle aux peintures fantastiques, après son passage au milieu de la cour des prostituées, il est prêt pour faire la découverte de l'origine érotique de la vie. Les colonnes se mettent à tourner, il entend la musique des sphères, il frissonne «d'extase ${ }^{37}$ », écrit Flaubert dans l'un des brouillons ; et il ajoute : "Tout semblait emporté dans un rythme aérien, il sentait ruisseler l'âme de Tanit. Elle le pénétrait, le dissolvait ${ }^{38}{ }^{3}$. Mais l'expérience est ambiguë :

Chaleur - quelque chose d'inexplicable, une mollesse s'échappait... comme si l'âme de Tanit, telle qu'un parfum plus subtil eût flotté dans l'air - se fût épandu dans l'air. Mâtho suffoquait - un tourment <malaise> indicible et pourtant le tourmentait - excitation <irritation> indicible où tourbillonnait le désir de Salammbô se dégageait de cette irritation <religion> générale. Il se dissolvait dans la tristesse d'un rêve <désir> infini et comme dans la force d'un élément. Tous ces symboles de la fécondation, de la mort et du mystère l'enivraient. Mâtho à travers les émerveillements [et les appréhensions] mystiques il songeait à Salammbô - elle se confondait avec la déesse elle-même et l'amour [le désir de sa personne] s'en dégageait plus fort [de cette religion] et plus haut comme les grandes fleurs qui s'élevaient du sein des eaux profondes ${ }^{39}$.

On voit l'hésitation entre excitation et irritation, irritation et religion. La sensualité se dégage des

${ }^{36}$ N.a.fr. $23658, f^{\circ} 447$.

${ }^{37}$ N.a.fr. $23658, f^{\circ} 452 v^{\circ}$.

${ }^{38}$ N.a.fr. $23658, \mathrm{f}^{\circ} 452 \mathrm{v}^{\circ}$

${ }^{39}$ N.a.fr. 23658, $\mathrm{f}^{\circ} 445$. Les soufflets indiquent des ajouts interlinéaires non biffés, les crochets des passages biffés. 
symboles religieux qui unissent la vie et la mort. Tanit est alors un «élément » insaisissable, une force de vie et de mort, invisible et répandue partout.

Dans un autre brouillon, Flaubert développe encore l'extase érotico-mystique : Mâtho est «dissout par les forces mystiques », sa personnalité s'en va "dans l'excès d'une sensation surhumaine », il est à la fois «terrifié » et «ravi ». Il se sentait, écrit Flaubert, « dévoré par les bêtes », mais en même temps «les couleurs le pénétraient, les griffes le déchiraient - brûlé, l'harmonie l'emportait [...] son être se dissolvait sous les effluves de la déesse et dans un spasme d'une volupté éternelle il s'écria qu'il allait mourir ${ }^{40} \gg$. Le Barbare ressemble à un saint Antoine, il se sent assailli par les bêtes peintes sur les murs du temple, tandis que l'ermite de 1874 croira vivre les choses abominables qui étaient peintes dans le tombeau du Pharaon où il avait trouvé refuge autrefois. Mais l'hallucination de Mâtho tourne à l'expérience érotique: elle est à la fois épouvantable et délicieuse. Flaubert ne laisse aucun doute dans ce brouillon sur la nature sexuelle de ce qu'il éprouve. La scène est le pendant du rêve de Salammbô qui sentait un dieu s'allonger sur elle dans le chapitre III. Mâtho, à son tour, croit s'unir à une puissance supérieure, qui est en l'occurrence la force érotique elle-même, la force qui a créé le monde et qui l'anime.

Les brouillons redoublent cette extase d'une seconde émotion forte au moment où Mâtho enfile le voile de Tanit dans la troisième et dernière salle. Comme le bouclier d'Achille qui est dans l'Iliade l'œuvre d'une pensée savante et qui représente le ciel, la mer, la terre, les dieux et les hommes, dans les brouillons de Salammbô le voile est une image de totalité. On y voit des animaux déjà aperçus sur les murs (une représentation de la création) ainsi que les Dieux principaux : Eschmoun, Melkarth et les Cabires. Par ses couleurs il est à la fois la nuit, le soleil et l'aurore. Au moment où il passe sa tête par l'encolure du zaïmph Mâtho éprouve un étourdissement, puis il est envahi par la force de la nature. Il vit une expérience panthéiste : il est habité par la puissance de l'univers et par celle des Dieux :

Il éprouve alors un étourdissement. Il reste immobile, silencieux, se passe la main sur le front plusieurs fois comme un homme étourdi par un coup - sourire - orgueil - Je n'ai plus rien à craindre - Ah je suis plus qu'un homme. Je sens la puissance des dieux en moi. Qui peut me résister? Je suis plus grand, plus fort, plus beau. Il me semble que je pourrai traverser les flammes, voler dans l'air, marcher sur les flots. Une joie céleste m'enivre! toutes les forces que nous avons vues je les sens rugir dans ma chair. J'ai des clartés dans la tête. Un élan m'enlève ${ }^{41}$.

Dans La Tentation de 1874, l'attaque traditionnelle des bêtes qui assaillent le saint selon la tradition hagiographique se transformera en une expérience panthéiste de fusion avec la matière vivante. Comme Mâtho Antoine se sentira habité par les forces du monde et dans un élan il se projettera dans la vie animale et dans le mouvement de la matière.

Dans le texte définitif de Salammbô, Flaubert ne modifie pas la scénographie de la seconde salle, la salle aux peintures fantastiques. Il conserve sa forme circulaire, les douze globes, le char. Mais il supprime l'idée centrale de l'épisode (Tanit résume en elle toutes les divinités étrangères) ainsi que toutes les références explicites à des déesses ou des attributs trop lisibles comme les lions du char de Cybèle. L'étrangeté provient alors d'une juxtaposition d'images déconnectées de leur contexte mythologique, de leur légende. Des symboles Flaubert conserve souvent l'image sans les significations. Par exemple de la nature cabirique de Tanit, glosée dans les brouillons, il reste dans

\footnotetext{
${ }^{40}$ N.a.fr. $23658, \mathrm{f}^{\circ} 470$.

${ }^{41}$ N.a.fr. $23658, \mathrm{f}^{\circ} 458 \mathrm{r}^{\circ}$.
} 
le texte définitif un étrange bonnet sur une statue (mais le lecteur peut ne pas connaître ce symbole des Cabires), douze globes azuréens dans une autre salle (le texte n'explique pas qu'ils sont les symboles des planètes, liées aux douze signes du zodiaque ${ }^{42}$ ), le feu tellurique enfin dans la dernière chambre, mais rien n'aide le lecteur qui n'aurait lu ni Creuzer ni Héraclite. Ce lecteur ne saurait pas que le feu peut être le symbole d'une force créatrice et il pourrait donc se demander comment expliquer la présence du feu de Moloch sur l'autel de Tanit.

L'étrangeté provient aussi de ce qu'Alfred Maury a théorisé comme un oubli de l'origine des métaphores. L'une des grandes thèses de l'historien des religions depuis les années 1840, et bien avant Max Müller, c'est que les mythes religieux proviennent d'une maladie du langage. Les premiers hommes produisaient des métaphores pour désigner les phénomènes naturels parce que leur langage et leur intelligence étaient inaptes à l'abstraction. Ils finirent par oublier qu'il s'agissait de métaphores et prirent alors au premier degré le sens figuré. C'est l'origine des dieux, et les légendes qui se développèrent ensuite eurent pour fonction d'expliquer des noms dont on avait oublié le sens figuré ${ }^{43}$. Flaubert utilise cette théorie non pour critiquer les légendes et symboles religieux comme le fit Alfred Maury mais pour en recréer. Dans un brouillon, il notait : « le ciel figuré par une femme dont le corps allongé s'étend en forme de voûte au-dessus de la terre ${ }^{44} »$. C'est l'origine de l'étrange peinture noire de la première salle : une femme qui couvre tout le plafond représente Tanit. On compare la nuit à un manteau qui tombe sur le monde, et ce manteau devient un zaïmph sacré et vénéré.

Une seconde thèse de Maury fournit aussi à Flaubert des principes d'invention mythologique. Dans les années 1850, l'historien des religions, féru d'études médicopsychologiques, avait pratiqué une critique positiviste des phénomènes extatiques, dévoilant une sensualité réprimée à l'origine de bien des extases et des visions chrétiennes. Flaubert, faisant le trajet inverse, réinvente un culte et des péripéties romanesques à partir de l'idée d'un mysticisme sensuel : un culte de la fécondité et le désir d'union mystique de Mâtho (et de Salammbô). La désacralisation positiviste qui, dans les travaux de Maury, peut bien passer pour une désymbolisation produit dans le roman un effet opposé. Maury explique comment naissent les symboles pour délivrer ses lecteurs des superstitions en leur montrant l'origine purement humaine et pathologique de bien des légendes chrétiennes. Flaubert utilise la méthode pour créer une cosmogonie fictionnelle étrange et génératrice de fantastique. Si le désir sexuel est au cœur de la religion de Tanit telle que la représente Flaubert, le dévoilement ne détruit pas les symboles. Au contraire, Flaubert multiplie les symboles en faisant des emprunts à diverses religions qui ont produit des représentations de divinités féminines, maternelles, ou érotiques. Maury désymbolise. Flaubert allie désymbolisation et resymbolisation : le roman a bien une dimension critique, mais le fond sexuel de la religion qui se confond avec le principe vital du monde est resymbolisé et produit une profusion d'images étranges.

La méthode de Flaubert ne ressemble guère à celle des historiens des religions qui qui construisent des rapprochements syncrétistes pour dégager des symboles les plus étranges une

\footnotetext{
${ }^{42}$ Creuzer, Religions de l'antiquité II, 1, p. 364. Mais il le dit à propos du culte de Cronos.

${ }^{43}$ C'est la thèse générale qu'Alfred Maury développe dans son Essai sur les légendes pieuses du moyenâge (Ladrange, 1843) en s'appuyant sur des analyses philologiques, dont on peut penser qu'elles inspireront celles de Max Müller. Celui-ci le citera dans le chapitre II de ses Essais sur la mythologie comparée («Mythologie grecque ») avec un certain nombre de spécialistes de la mythologie grecque. Mais il limite son importance en signalant qu'Alfred Maury n'avait lui-même pas la prétention d'ajouter beaucoup de conclusions nouvelles et personnelles à ce qui était déjà connu sur les différents sujets dont il traite. L'ouvrage de Müller sera traduit en français et publié avec une préface de Renan en 1859.

${ }^{44}$ N.a.fr. $23658, \mathrm{f}^{\circ} 415$.
} 
signification et montrer leur formation ou leur évolution dans l'histoire par des échanges culturels et syncrétistes. Au contraire, des brouillons au texte, le travail de Flaubert opacifie la signification des mythes et des symboles. Il retourne la méthode de Creuzer : loin d'éclairer les mythes les uns par les autres, il fait des trous dans les récits mythiques, il procède par effacement de significations, dispersion ou suppression des symboles trop lisibles, par juxtaposition d'images plastiques évidées de leur signification, ou il juxtapose des représentations irréductiblement contradictoires : dans le chapitre V Tanit est encore «la dernière inventée » comme le disait Schahabarim, et en même temps l'Origine de tout, et des dieux mêmes sous le regard de Mâtho. La rationalisation des divergences par la focalisation ne réduit pas totalement les contradictions.

L'étrangeté tient aussi à la suppression des références explicites - ou trop claires pour un lecteur cultivé - à des divinités bien connues comme Artémis, Cybèle, Minerve ou Vénus. Tanit perd les dauphins de Vénus, la gorgone de Minerve, la tour de Cybèle, et son char désormais d'ivoire n'est plus attelé aux lions de la Grande déesse de Phrygie. La phrase qui explicitait sa dimension syncrétiste et les emprunts aux autres religions barbares disparaît également :

Des écailles, des plumes, des fleurs et des oiseaux lui montaient jusqu'au ventre. Pour pendants d'oreilles elle avait des cymbales d'argent qui lui battaient sur les joues. Ses grands yeux fixes vous regardaient ; une pierre lumineuse, enchâssée à son front dans un symbole obscène, éclairait toute la salle, en se reflétant au-dessus de la porte, sur des miroirs de cuivre rouge ${ }^{45}$.

Mais le sens des emblèmes supprimés se diffuse dans le texte. Tanit est bien une déesse sensuelle comme Vénus, panthée comme Artémis, une déesse Nature comme Cybèle, mère comme elle, mais aussi castratrice comme celle-ci (Mâtho mourra comme Attis), et terrible comme la Minerve à la gorgone. Elle porte étrangement comme un troisième œil un symbole obscène qui n'est pas décrit, et qui peut faire rêver le lecteur à certaines idoles indiennes. Dans la dernière salle, le Zaimph est déposé sur un cône, dont Creuzer nous apprend la signification sexuelle : Vénus était représentée par un cône à Paphos, et on peut rapporter, nous dit Creuzer, cette forme symbolique au culte antique du phallus ou de l'Yoni-lingam, "c'est-à-dire des forces productrices de la nature dans leur union féconde », culte représenté en Inde et en Égypte ${ }^{46}$. Dans le texte de Flaubert, la statue de Tanit au symbole obscène demeure dans son étrangeté, et le symbolisme du cône dans la troisième salle n'est pas davantage glosé. Toutefois si le symbole de l'Yoni-lingam, emblème érotique de l'union mystique ${ }^{47}$, n'est pas nommé - ce qui ne permet guère au lecteur peu familier de Creuzer d'identifier exactement le « symbole obscène » dont il est question dans le roman - il n'est peut-être pas étranger au brusque désir qui saisit Mâtho d'aller rejoindre Salammbô au moment s'enveloppe du voile de la déesse devant le cône. L'effacement des significations, la dispersion ou la disparition des emblèmes, des symboles, des indices, sont les procédés qui créent autour des représentations un halo d'incertitude et donnent au texte une profondeur symbolique. Bien qu'effacé, le sens des symboles continue à agir dans le texte romanesque, générant des sentiments et des péripéties.

45 Flaubert, Salammbô 138.

${ }^{46}$ Creuzer, Religions de l'antiquité II, 1, p. 222. Il signale aussi qu'un cône immense aurait été retrouvé dans les ruines de Carthage (Ibid., p. 235).

${ }^{47}$ Dans Religions de l'antiquité, on apprend qu'il représente les organes de la génération (I, p. 149) et qu'il est évoqué comme une colonne de feu dans une coupe féconde (I, 1, p. 158). 
$* * *$

Entre La Tentation de 1849 (ou celle de 1856) et le dénouement de La Tentation de 1874 qui invente un nouveau mythe de l'origine de la vie en retravaillant les représentations d'époque $^{48}$, Salammbô constitue une étape intermédiaire. Avec Carthage, Flaubert traite un ensemble de croyances puniques et phéniciennes dont Creuzer avait déjà montré le syncrétisme par rapport aux grandes religions de l'antiquité ${ }^{49}$. Il choisit surtout une cosmogonie qui peut être interprétée en un sens matérialiste, ce que firent avant lui Maury (dans ses notes à l'ouvrage de Creuzer) ainsi que certains érudits allemands cités par Renan. Le premier indique que, selon Sanchionathon, c'est de la matière que "procéda toute semence de création ${ }^{50}$. Le second trouve dans les écrits attribués à Sanchionathon une théorie de la génération spontanée $e^{51}$ et il cite une série de travaux érudits qui y ont trouvé une philosophie de la «matière première » 52 . Prudent, Renan estime toutefois que ces conjectures sont dépourvues de l'autorité des manuscrits. Mais on sait que le romancier pour sa part n'éprouve pas les mêmes nécessités surtout lorsqu'il s'agit d'inventer des croyances.

Pour élaborer les cosmogonies des chapitres III et V Flaubert fragmente et condense des mythes, des symboles et des idées empruntés à des ouvrages qui traitent de l'antiquité. Mais il privilégie les éléments qui peuvent infléchir la représentation vers une mythologie du vivant. L'étrangeté du temple de Tanit tient à une profusion des images, à l'impression - que ressent Mâtho - de toucher à une origine du monde, de la vie. Peu importe que le personnage superstitieux porte encore au crédit de Tanit la force qu'il éprouve dans le temple. Dans son parcours de salle en salle, jusqu'au saint des saints, Flaubert lui fait remonter la chaîne des formes qui incarnent la vie (les prostituées sacrées puis les animaux), jusqu'au symbole conique, abstrait et désincarné, sur lequel est posé le voile et devant lequel brûle le feu héraclitéen, principe de toute chose, force universelle. Mais, comme Salammbô, Mâtho a besoin de symboles, de croyances, et de formes anthropomorphiques. Quoi qu'il en soit, en dépit de la superstition du personnage focalisateur, Flaubert, pour sa part, invente une nouvelle mythologie, entre antiquité et modernité. Elle fusionne des symboles religieux anciens et quelques idées ou images qui pouvaient recevoir une interprétation plus moderne, dans un contexte

${ }^{48}$ Flaubert a l'idée d'écrire un nouveau dénouement en lisant La Création de Quinet (1870) qu'il cite dans l'un des scénarios de l'œuvre (N.a.fr 23671, $\mathrm{f}^{\circ} 78 \mathrm{v}^{\circ}$ ) : (v. Quinet. La creation t 1er 38-39) : " après le sphinx $\alpha$ la Chimère, les animaux antediluviens, informes / $\alpha$ peu à peu arriver, par une serie de monstruosités (symboliques) / à la cellule vivante, à l'Etre, à la matière. » Un autre folio porte la trace d'une découverte de Haëckel, la monère (f 226 ), dans Histoire de la création des êtres organisés est paru en allemand en 1868 puis en français en 1874. Flaubert a lu également La Mer de Michelet (1861).

${ }^{49}$ Renan, pour sa part, estime que toutes les cosmogonies sémitiques conservées qu'elles soient phéniciennes, babyloniennes ou bibliques sont «calquées sur le même plan et de même famille » (Ernest Renan, Mémoire sur l'origine et le caractère véritable de l'histoire phénicienne qui porte le nom de Sanchoniathon [Paris : Imprimerie impériale, 1858] 250).

${ }^{50}$ Creuzer, Religions de l'antiquité, notes du livre IV, p. 860.

${ }^{51}$ Renan, Mémoire 253.

${ }^{52}$ Renan, Mémoire 254. Sur ce point voir 1'article d'Agnès Bouvier, « Au fracas de la foudre, les animaux intelligents s'éveillèrent. De la Genèse » de Salammbô à la théorie de la génération spontanée », Flaubert [En ligne], 5 |2011, mis en ligne le 12 juillet 2011, consulté le 29 août 2013. URL : http://flaubert.revues.org/1323. 
épistémologique où les savoirs du vivant sont en débat.

Université Paris-Est Marne-la-Vallée et Fondation Maison des sciences de l'Homme de Paris 